\title{
Degradation of difluorobenzenes by the wild strain Labrys portucalensis
}

I. S. Moreira ${ }^{1}$, M. F. Carvalho ${ }^{1}$, C. L. Amorim¹, D. B. Janssen², P. M. L. Castro

${ }^{1}$ Escola Superior de Biotecnologia, Universidade Católica Portuguesa, Porto, Portugal

2 Groningen Biomolecular Sciences and Biotechnology Institute, University of Groningen, Groningen, The Netherlands

\section{Methods}

\section{Introduction}

Haloaromatic compounds have been produced industrially on a large scale for several decades, becoming environmental pollutants of soil, water and air. Fluorinated are among these, because of their useful properties, which make them suitable for a wide range of applications [1]. Whereas the degradation of chlorinated and brominated compounds has been studied extensively, fluoroaromatics have received less attention and fewer studies are available on their biodegradation. This study focuses on the biodegradation of difluorobenzenes (DFBs), which are commonly employed as chemical intermediates in various pharmaceutical and agricultural applications. Complete biodegradation of DFBs has not yet been reported to the best of our knowledge.

A previously isolated microbial strain (strain F11), identified as Labrys portucalensis [2], and with the capacity to degrade fluorobenzene (FB) as sole carbon and energy source, was tested for its capability to degrade 1,2-, 1,3- and 1,4difluorobenzene.

\section{Results}

\section{DFBs biodegradation as a sole carbon and energy source}

1,3-DFB was completely degraded and a stoichiometric liberation of fluoride was observed in 20 days (Fig. 1). L. portucalensis F11 was not able to defluorinate 1,2- and 1,4-

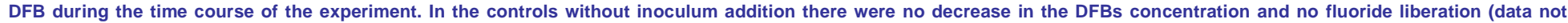
shown).
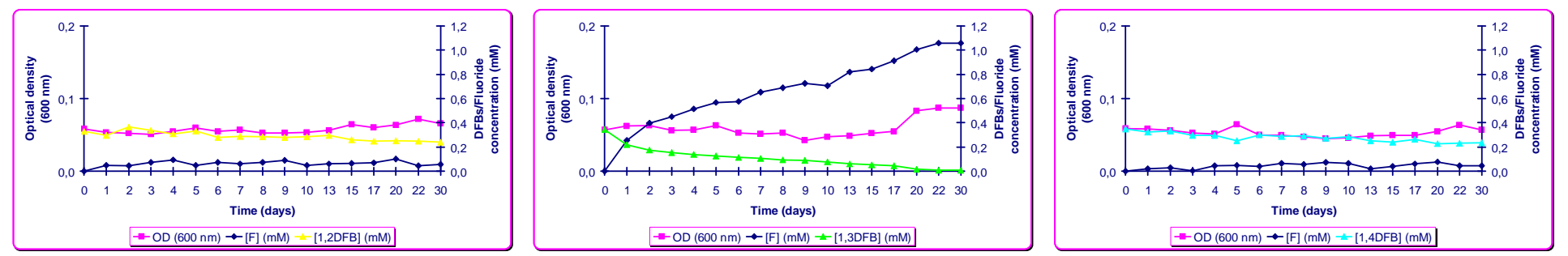

Figure 1. Optical density (OD $600 \mathrm{~nm}$ ), DFBs concentration and fluoride release along time (days) of F11 cultures pregrown on FB and supplemented with 0.5 mM of 1,2-DFB, 1,3-DFB or 1,4-DFB. The experiment was conducted in triplicate.

\section{DFBs biodegradation in co-metabolism with FB}

The highest growth was observed in cultures fed with $0.5 \mathrm{mM}$ of 1,3-DFB and $1 \mathrm{mM}$ of FB (Fig. 2), with strain F11 being capable to degrade these compounds in less than two

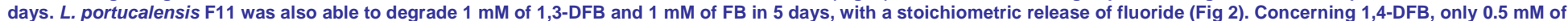

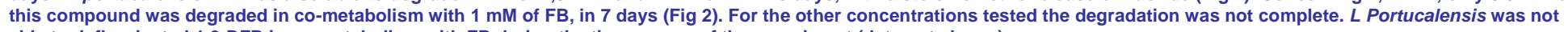
able to defluorinated 1,2-DFB in co-metabolism with FB during the time course of the experiment (data not shown).
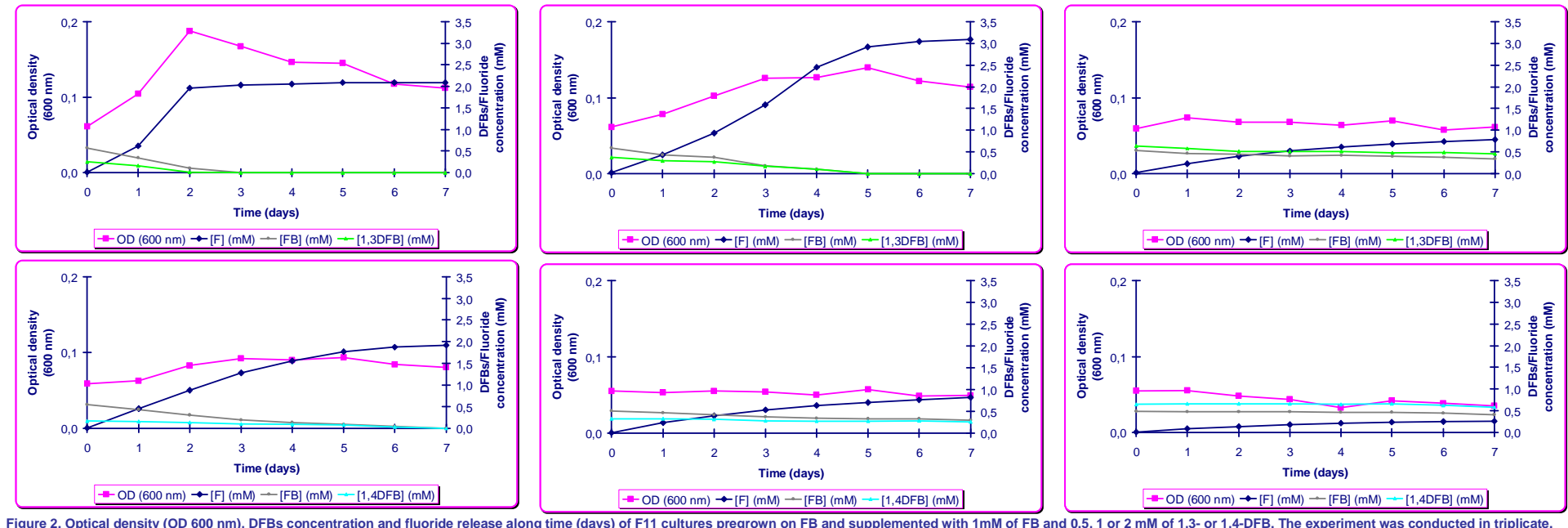

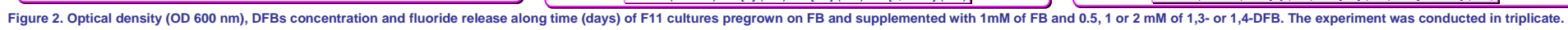

\section{References}

[1] Key B, Howell R and Criddle C. (1997). "Fluorinated organics in the biosphere". American Chemical Society. 31:2445-2454.

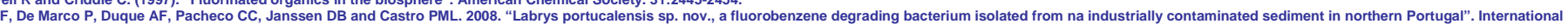

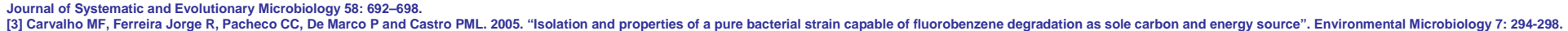
[3] Carvalho MF, Ferreira Jorge R, Pacheco CC, De Marco P and Castro PML. 2005. "Isolation and properties of a pure bacterial strain capable of fluorobenzene degradation as sole carbon and energy source". Environmental Micro
[4] Carvalho MF, Alves CCT, Ferreira MIM, De Marco P and Castro PML. 2002. "Isolation and initial characterization of a bacterial consortium able to mineralize fluorobenezene". Applied and Environmental Microbiology 68: 102-105. Acknowledgements 